\title{
Attachment Behavior of Fission Products to Solution Aerosol
}

\author{
Koichi Takamiya*, Toru Tanaka, Shinnosuke Nitta, Satoshi Itosu, Shun Sekimoto, Yuichi Oki, Tsutomu Ohtsuki \\ Research Reactor Institute, Kyoto University, Osaka, Japan
}

\section{Original Research}

Received July 17, 2015

Revision September 20, 2016

Accepted September 22, 2016

Corresponding author: Koichi Takamiya

Research Reactor Institute, Kyoto University, 1010 Asashiro-Nishi-2, Kumatori, Sen-nan-gun, Osaka, Japan 590-0494

Tel: +81-724-51-2460,

Fax: +81-724-51-2632

E-mail: takamiya@rri.kyoto-u.ac.jp

This is an Open-Access article distributed under the terms of the Creative Commons Attribution NonCommercial License (http://creativecommons.org/ licenses/by-nc/4.0) which permits unrestricted noncommercial use, distribution, and reproduction in any medium, provided the original work is properly cited.

Copyright $\odot 2016$ The Korean Association for Radiation Protection

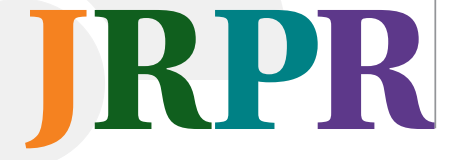

Background: Various characteristics such as size distribution, chemical component and radioactivity have been analyzed for radioactive aerosols released from Fukushima Daiichi Nuclear Power Plant. Measured results for radioactive aerosols suggest that the potential transport medium for radioactive cesium was non-sea-salt sulfate. This result indicates that cesium isotopes would preferentially attach with sulfate compounds. In the present work the attachment behavior of fission products to aqueous solution aerosols of sodium salts has been studied using a generation system of solution aerosols and spontaneous fission source of ${ }^{248} \mathrm{Cm}$.

Materials and Methods: Attachment ratios of fission products to the solution aerosols were compared among the aerosols generated by different solutions of sodium salt.

Results and Discussion: A significant difference according as a solute of solution aerosols was found in the attachment behavior.

Conclusion: The present results suggest the existence of chemical effects in the attachment behavior of fission products to solution aerosols.

Keywords: Radioactive aerosol, Solution aerosol, Attachment behavior, Attachment ratio, Fission product

\section{Introduction}

Radioactive aerosols were released from Fukushima Daiichi Nuclear Power Plant and spread in the environment. The radioactive aerosols have been collected and measured for their activity, size distribution, chemical composition and others up to date. In order to identify transport mediums for radioactive materials, both activity size distributions of radioactive isotopes and mass size distributions of aerosol species were measured [1-6]. As the results, it was found that sulfate compounds were the potential transport medium for radioactive cesium isotopes. This result suggests that cesium isotopes would preferentially attach with sulfate compounds. But there is no report about such selectivity for other radioisotopes.

In the present work the attachment behavior of fission products on aqueous solution aerosols of sodium salts has been studied using a generation system of solution aerosols and spontaneous fission source of ${ }^{248} \mathrm{Cm}$. The solution aerosols were generated by three kinds of aqueous solution of sodium salts of chloride, bromide and iodide. Attachment ratios of fission products to the solution aerosols were compared among the solution aerosols of different sodium salts in order to elucidate a roll of chemical effects 
in the attachment behavior of fission-product to solution aerosols.

\section{Materials and Methods}

Solution aerosols were generated using a combined system of an atomizer 3076 (TSI, Shoreview, MN), furnace, diffusion dryer, neutralizer as shown in Figure 1. Various concentrations of aqueous solutions of sodium chloride, sodium bromide and sodium iodide (Wako, Osaka, Japan) were used as raw materials in the atomizer. Nitrogen gas was supplied at pressure of $2.5 \mathrm{~kg} \cdot \mathrm{cm}^{-2}$ to the atomizer to discharge solution aerosols. The aerosols were passed through the furnace, which was kept constant at $80^{\circ} \mathrm{C}$ and the diffusion dryer with an infill of silica gel sequentially to be a primary solution aerosol. Particle size distributions of the primary aerosols ejected from the diffusion dryer were measured using a scanning mobility particle sizer (SMPS) spectrometer. The SMPS spectrometer was composed by an electrostatic classifier 3080L (TSI, Shoreview, MN) and condensation particle counter 3025A (TSI, Shoreview, MN) to measure the size distribution of submicron range. The primary aerosols were injected into the fission product chamber in which ${ }^{248} \mathrm{Cm}$ spontaneous fission source covered with a Havar foil of 2.5 $\mu \mathrm{m}$ thickness was placed. The activity of ${ }^{248} \mathrm{Cm}$ which undergoes spontaneous fission with a branching ratio of $8.39 \%$ [7] was $40 \mathrm{kBq}$ that corresponds to emissions of about three thousand fission products per second in the chamber. Some of fission products emitted from the ${ }^{248} \mathrm{Cm}$ source attached to the primary aerosol particles, and then radioactive aerosol was generated. The radioactive aerosol was ejected from the chamber and collided against a greased filter paper to be collected for thirty minutes. The collection time of thirty minutes is suitable for measuring gamma-rays emitted from fission products which half-life is a few tens of minutes. After seven minutes from the end of the collection, gamma-ray spectrometry for the filter was performed using Ge-detector

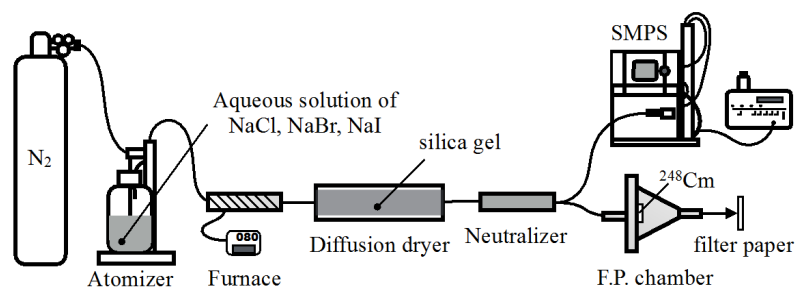

Fig. 1. Experimental setup for generation of radioactive solution aerosols and measurement of particle size distribution.
GC4020 (Canberra, Meriden, CT). The gamma-ray measurements for ten minutes were repeated six times continuously. Whole fission fragments emitted in the chamber from the ${ }^{248} \mathrm{Cm}$ source for thirty minutes was also measured by the following manner; a greased filter paper was located above the source for thirty minutes to collect whole fission products and after seven minutes of the collection the gamma-ray spectrometry was carried out using the same routine as the radioactive aerosol sample. Four nuclides of ${ }^{104} \mathrm{Tc}\left(T_{1 / 2} 18.3\right.$ $\mathrm{min}),{ }^{107} \mathrm{Rh}\left(T_{1 / 2} 21.7 \mathrm{~min}\right),{ }^{131} \mathrm{Te}\left(T_{1 / 2} 25.0 \mathrm{~min}\right)$, and ${ }^{134} \mathrm{I}\left(T_{1 / 2}\right.$ $52.5 \mathrm{~min}$ ) were observed in the all experiments.

\section{Results and Discussion}

Size distributions of the primary solution aerosols generated by the present system using various concentrations of each sodium salt solution have been measured by the SMPS spectrometer. A log-normal size distribution was observed for each measurement. The sizes of particles observed in the present experiments were in the range from 15 to $660 \mathrm{~nm}$, which is a measurable range of the present setup of the SMPS spectrometer. The geometric mean diameters (GMDs) of the primary aerosols range from 40 to $100 \mathrm{~nm}$, and the GMD increases as the concentration of the raw solutions in the atomizer increases. The geometric standard deviations range from 0.54 to 0.58 for all primary aerosols. Total surface area of the aerosol particles through the aerosol collection was estimated for each primary aerosol on the basis of the measured size distribution assuming that the solution aerosol were spherical in shape. Attachment behavior of fission products is considered in the following discussion correlating with the total surface area.

An attachment ratio of fission products to the aerosol particles is used as an index to valuate the attachment behavior. A ratio of fission products that attached to the aerosol particles was estimated by $\mathrm{C}_{\text {aerosol }} / \mathrm{C}_{\text {all, }}$, where $\mathrm{C}_{\text {aerosol }}$ and $\mathrm{C}_{\text {all }}$ are net counts of photopeaks in the gamma-ray spectra measured for the radioactive aerosol and all fission products emitted from ${ }^{248} \mathrm{Cm}$, respectively. The net counts of photopeaks were calculated by Covell's method [8]. The attachment ratio for individual fission product could be estimated for four observed nuclides. Figure 2 shows the relationship between the attachment ratios of four fission products $\left({ }^{104} \mathrm{Tc},{ }^{107} \mathrm{Rh},{ }^{131} \mathrm{Te}\right.$, and ${ }^{134} \mathrm{I}$ ) and the total surface area of the primary aerosol. Squares, triangles and circles indicate the attachment ratios for primary solution aerosols of sodium chloride, sodium 


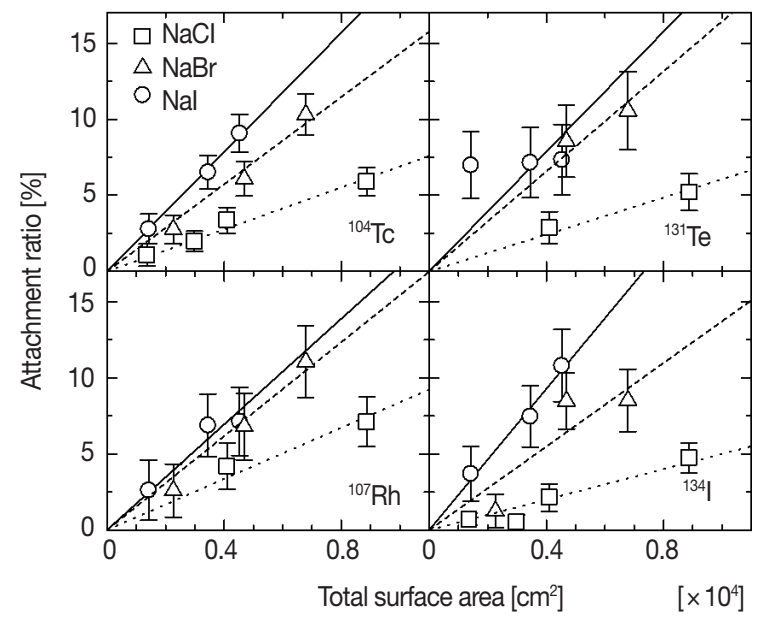

Fig. 2. Variation of the attachment ratio of each fission product as a function of total surface area of the primary solution aerosol particles.

bromide and sodium iodide, respectively. Error values were estimated on the basis of statistic errors (one standard deviation) in the gamma-ray spectrometry. The similar tendency of amplitudes of the attachment ratio among three kinds of solutes is found, namely the ratio for iodide is the largest and that for chloride is the smallest at the same total surface area. This difference of attachment ratios among the solutes means that four kinds of fission products observed in the present experiment behave differently against the solution aerosols composed by the different solute in generating process of radioactive aerosols.

The attachment ratios in Figure 2 seem to be proportionate to the total surface area except for ${ }^{131} \mathrm{Te}$ to sodium iodide. The proportional relation would be attributable to ratio of physical collision between fission products and primary aerosol particles. Each relation was fitted with linear function based on a hypothesis that the physical collision is predominant in the attachment behavior of fission products. Because the proportionate increase does not appear in attachment ratios of ${ }^{131} \mathrm{Te}$ to sodium iodide, the dominant attachment process might not be the physical collision but other process. But detailed analysis is difficult because of large error values for the ratio of ${ }^{131} \mathrm{Te}$ in the present work. The results of the fitting are shown by three lines for each fission product in Figure 2. Dotted, dashed and solid lines in the figure indicate the fitted results for chloride, bromide and iodide, respectively. The slopes of fitted lines for four kinds of fission products are shown according to three solutes in Figure 3. Symbols of circle, square, diamond and triangle show the slope

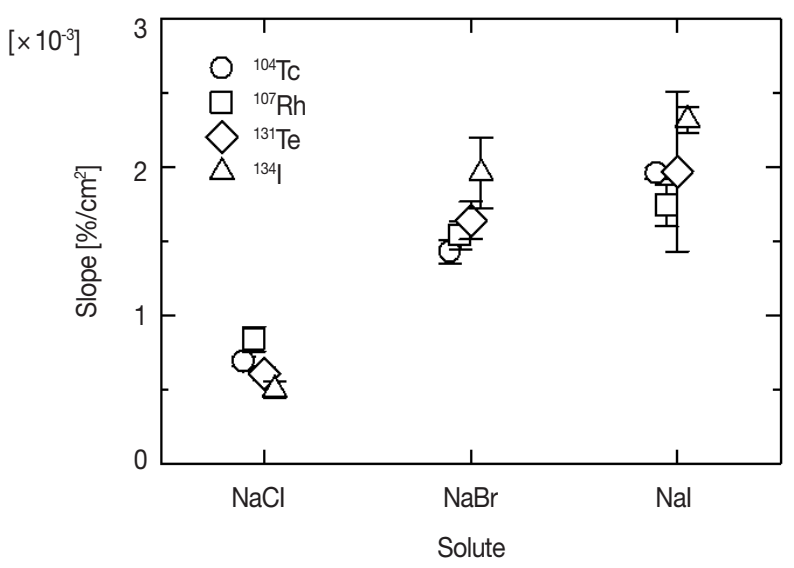

Fig. 3. Constant of proportionality between the attachment ratio and the total surface area of the primary solution aerosol particles for each fission product and the solute of solution aerosol.

for ${ }^{104} \mathrm{Tc},{ }^{107} \mathrm{Rh},{ }^{131} \mathrm{Te}$, and ${ }^{134} \mathrm{I}$. The error values shown by vertical bars indicate fitting errors. There is not a significant difference among the fission products but a distinct difference between the slopes of sodium chloride and other two solutes. In the generation process of radioactive aerosols, the attachment ratio is presumed to be proportionate to a geometric collision ratio, namely surface area of an aerosol particle. And if chemical state of an aerosol particle and/or an attaching fission product affects the attachment behavior, the constant of proportion would vary according as the chemical state. The difference of the slopes according to the solute shown in the present experiment suggests the existence of chemical effect in the attachment behavior. One of the origins of the difference of the chemical effect would be density profiles of the anions in solution aerosol particles. Molecular dynamics simulations performed by L. Tuma et al. reveals a propensity of halide anions for the water/vapor interface, which increases in the series $\mathrm{Cl}^{-}<\mathrm{Br}^{-}<\mathrm{I}^{-}$[9]. The propensity would cause differences of attracting force between halide ions in a solution aerosol particle and fission product ions in the nitrogen gas phase.

\section{Conclusion}

Attachment behavior of fission products to solution aerosols generated from aqueous solutions of sodium chloride, sodium bromide and sodium iodide has been observed. Attachment ratios of fission products emitted from ${ }^{248} \mathrm{Cm}$ source have been estimated by gamma-ray measurements 
for all fission products emitted from the source and fission products attaching to the aerosol particles. It was found that proportional relationship exists between the attachment ratios and the total surface area except for ${ }^{131} \mathrm{Te}$ to the solution aerosol of sodium iodide. In comparison of slopes of the relationship, there found a significant difference according as a solute of solution aerosols. The difference according to the solute shown in the present work suggests the existence of chemical effect in the attachment behavior of fission products to solution aerosols.

\section{Acknowledgements}

This work was supported by JSPS KAKENHI Grant Numbers 24110005 and 26286076 .

\section{References}

1. Kaneyasu N, Ohashi H, Suzuki F, Okuda T, Ikemori F. Sulfate aerosol as a potential transport medium of radiocesium from the Fukushima nuclear accident. Environ. Sci. Technol. 2012;46 (11):5720-5726.

2. Miyamoto Y, Yasuda K, Magara M. Size distribution of radioac- tive particles collected at Tokai, Japan 6 days after the nuclear accident. J. Environ. Radioact. 2014;132:1-7.

3. Doi T, Masumoto K, Toyoda A, Tanaka A, Shibata Y, Hirose K. Anthropogenic radionuclides in the atmosphere observed at Tsukuba: characteristics of the radionuclides derived from $\mathrm{Fu}$ kushima. J. Environ. Radioact. 2013;122:55-62.

4. Malá H, Rulík P, Becková V, Mihalík J, Slezáková M. Particle size distribution of radioactive aerosols after the Fukushima and the Chernobyl accidents. J. Environ. Radioact. 2013;126:92-98.

5. Masson O, Ringer W, Malá H, Rulik P, Dlugosz-Lisiecka M, Eleftheriadis K, Meisenberg O, De Vismes-Ott A, Gensdarmes F. Size distributions of airborne radionuclides from the Fukushima nuclear accident at several places in Europe. Environ. Sci. Technol. 2013;47(19):10095-11003.

6. Muramatsu H, Kawasumi K, Kondo T, Matsuo K, Itoh S. Sizedistribution of airborne radioactive particles from the Fukushima accident. J. Radioanal. Nucl. Chem. 2015;303(2):1459-1463.

7. Martin, MJ. Nuclear Data Sheets for A = 248. Nucl. Data Sheets. 2014;122:377-409.

8. Covell DF. Determination of gamma-ray abundance directly from total absorption peak. Anal. Chem. 1959;31(11):1785-1790.

9. Tuma L, Jenicek D, Jungwirth P. Propensity of heavier halides for the water/vapor interface revisited using the Amoeba force field. Chem. Phys. Lett. 2005;411:70-74. 